OPEN ACCESS

Edited by:

Hai YuXu,

China Academy of Chinese

Medical Sciences, China

Reviewed by:

Xiaojun Wu,

Shanghai University of Traditional

Chinese Medicine, China

Zhe-Sheng Chen,

St. John's University, United States

*Correspondence:

Cheng Lu

cheng@163.com

Luqi Huang

hlq910@126.com

Specialty section:

This article was submitted to

Ethnopharmacology,

a section of the journal

Frontiers in Pharmacology

Received: 24 June 2020

Accepted: 14 August 2020

Published: 15 September 2020

Citation:

Xiong Y, Li NX, Duan N, Liu B, Zhu H,

Zhang C, Li L, Lu C and Huang L

(2020) Traditional Chinese Medicine in

Treating Influenza: From Basic

Science to Clinical Applications.

Front. Pharmacol. 11:575803.

doi: 10.3389/fphar.2020.575803

\section{Traditional Chinese Medicine in Treating Influenza: From Basic Science to Clinical Applications}

\author{
Yibai Xiong ${ }^{1}$, Na Xiao $L_{i}{ }^{1}$, Naifang Duan ${ }^{1}$, Bin Liu ${ }^{1}$, Hui Zhu ${ }^{2}$, Chi Zhang ${ }^{3}$, Li Li ${ }^{1}$, \\ Cheng $\mathrm{Lu}^{1 *}$ and Luqi Huang ${ }^{2 *}$ \\ 1 Institute of Basic Research in Clinical Medicine, China Academy of Chinese Medical Sciences, Beijing, China, ${ }^{2}$ China \\ Academy of Chinese Medical Sciences, Beijing, China, ${ }^{3}$ Dongzhimen Hospital, Beijing University of Chinese Medicine, \\ Beijing, China
}

Influenza infection is a highly contagious, acute febrile respiratory disease caused by the influenza virus. Traditional Chinese Medicine (TCM) has dominated plenty of theoretical and practical approaches in the treatment of influenza. It is, therefore, important to highlight the effects of TCM in the clinical treatment of influenza and their impact on inhibiting the growth of this virus in laboratory experiments. We scrutinized existing evidence on whether TCM is effective in clinical applications. Moreover, we described the potential mechanisms of TCM against the influenza virus. Our findings provide analytical evidence that supports the effectiveness of TCM in treating influenza infections as well as their mechanisms against this virus.

Keywords: influenza, Traditional Chinese Medicine, herbal medicine, influenza symptoms, anti-influenza virus, review

\section{INTRODUCTION}

Influenza is an acute viral respiratory infection that was first described in the 4th century BC. At the time, it was estimated to have caused nearly 60,000 mortalities in Venice, Italy. Due to the impact of this disease in Venice, it was understood by folklore to be a punishment from God and was therefore named "Influenza (Devil)" (JJ, 2010). The influenza virus belongs to the Orthomyxoviridae family. It is a singlestranded negative-sense RNA virus exhibiting an eight-segmented genetic material (Brody, 2019). Its genetic material is made up of Polymerase Basic Protein 1 (PB1), Polymerase Basic Protein 2 (PB2), Polymerase Acidic (PA), Hemagglutinin (HA), Nucleoprotein (NP), Neuraminidase (NA), Matrix Protein (M), and Nonstructural Protein (NS) (Lu, 2008). Depending on the composition of its NP and $\mathrm{M}$, this virus is grouped into three types (A, B, and C). Its subtypes are determined by the antigenicity of two surface glycoproteins, the 18 HA and 11 NA (Webster et al., 1992; Kawaoka, 2006; Huang et al., 2008). It is extremely susceptible to mutations because its RNA genome lacks proof-reading ability during replication as is the case with DNA genomes. Moreover, the mutations are also attributed to antigenic drifts. These mutating types propagate this virus, leading to epidemics in certain seasons (Cox

Abbreviations: TCM, Traditional Chinese Medicine; PB1, Polymerase Basic Protein 1; PB2, Polymerase Basic Protein 2; PA, Polymerase Acidic; HA, Hemagglutinin; NP, Nucleoprotein; M, Matrix Protein; NS, Nonstructural Protein; NA, Neuraminidase; NAIs, Neuraminidase Inhibitors; WHAFS, Wind-heat affecting Fei syndrome; RCT, Randomized Controlled Trial; HCP, Houttuynia cordata polysaccharide. 
and Subbarao, 2000; He et al., 2011; Kamali and Holodniy, 2013) and, notably, they hinder the development of new antiviral drugs. First-line antiviral drugs in the treatment of influenza rely on blocking the action of NP (Gaitonde et al., 2019). However, the side-effects of anti-NA drugs render many patients unavailable to treatment. In China, the broadly prescribed anti-influenza drugs include oseltamivir, zanamivir, and peramivir (Lee et al., 2017). Notably, Oseltamivir has been associated with resistance (Govorkova et al., 2001; Kiso et al., 2004; Moscona, 2004) and leads to immunocompromised conditions (Govorkova et al., 2001; Moscona, 2009; Panning, 2013). Zanamivir is administered through inhalation, and challenges over its use have been described (Shetty and Peek, 2012). Due to its limited bioavailability, peramivir can only be administered intravenously (Birnkrant and Cox, 2009). Therefore, these challenges call for more effort and attention toward developing fresh strategies to combat influenza. Previously, TCM demonstrated a positive anti-viral effect on influenza virus and alleviated the symptoms among patients (He et al., 2019; Nagai et al., 2019). In TCM theory, influenza disease was classified as "YI Disease" and was first reported in Huang Di Nei Jing. During the Han Dynasty, a theory of Shang Han (Treatise of Exogenous Febrile Diseases or Discourse on Cold-Damage Disorders) was written by Zhang Zhonging to treat influenza. Later, during the Ming and Qing Dynasties, the emergence of "Wen Yi Lun (Analysis of Epidemic Warm Diseases)" revealed that TCM had contributed enormously to the prevention and treatment of influenza for thousands of years. Despite studies showing that TCMs are effective against influenza (Moscona, 2004; Tian et al., 2011), further studies on the development of novel anti-influenza agents are essential (Rajasekaran et al., 2013; Chang et al., 2014; Ding et al., 2014; Li C. et al., 2015). Herein, we summarize the impacts of TCM in treating influenza from basic experiments to clinical applications.

\section{CONFIRMED IN CLINICAL APPLICATION}

In Asia and some countries in Europe, TCM has been proven to be effective and secure for treating influenza (Moscona, 2004). Traditional Chinese Medicine (TCM) subdivides influenza into cold syndrome and heat syndrome. The major symptoms of wind-cold type include severe cold, light heat, lack of sweating, headache, sore limbs, and stuffy nose. On the other hand, high fever, a mild cold, headache, sore throat, cough are the primary symptoms of wind-heat type. By relying on the concept of evidence-based medicine, several clinical trials have been conducted to verify the efficacy and safety of TCM.

\section{Meta-Analysis}

A meta-analysis enrolled 30 studies consisting of 3,444 cases in all to investigate efficacy and safety of TCM in the treatment of influenza infection. Our findings indicated that the mean time fever resolution of the TCM treatment group was statistically significant compared to the control group. Additionally, the synergistic effects of TCM and conventional medicines on viral infections were better compared to the control group (Li J. H. et al., 2016). Further, a different TCM prescription from Shang Han Lun, ma-huang-tang( $5 \mathrm{~g}$ of ephedra equisetina bunge, $5 \mathrm{~g}$ of prunus armeniaca L., $4 \mathrm{~g}$ of cinnamomum verum J.Presl, and $1.5 \mathrm{~g}$ of glycyrrhiza uralensis fisch. ex DC.) was confirmed that it alleviated fever when singly administered or in combination with neuraminidase inhibitors (NAIs) and might be a well-tolerated treatment as reported by a systematic review and meta-analysis conducted by Japanese scientists (Yoshino et al., 2019). Andrographis paniculata was indicated to be beneficial and safe for relieving influenza symptoms and shortening time to symptom resolution ( $\mathrm{Hu}$ et al., 2017).Conclusively, TCM are validated in lessening influenza symptoms, such as duration for defervescence and cough.

\section{Randomized Clinical Trial (RCT)}

Researchers conducted a randomized trial for comparing the efficacy and safety of oseltamivir and maxingshigan-yinqiaosan [6 $\mathrm{g}$ of honeyfried ephedra equisetina bunge; $10 \mathrm{~g}$ of anemarrhena asphodeloides bunge; $15 \mathrm{~g}$ of artemisia annua L.; $30 \mathrm{~g}$ of gypsum fibrosum; $15 \mathrm{~g}$ of lonicera japonica thunb.; $15 \mathrm{~g}$ of scutellaria baicalensis georgi; $15 \mathrm{~g}$ of stir-baked prunus armeniaca L.; $15 \mathrm{~g}$ of forsythia suspensa (thunb.) vahl; $6 \mathrm{~g}$ of mentha canadensis L.; $15 \mathrm{~g}$ of fritillaria thunbergii miq.; $15 \mathrm{~g}$ of arctium lappa L.; and $10 \mathrm{~g}$ of glycyrrhiza uralensis fisch. ex DC.] originating from Shang Han Lun and Wen Bing Tiao Bian, in treating uncomplicated influenza. Results have shown that singular use or combinations of oseltamivir and maxingshigan-yinqiaosan minimized defervescence time in patients diagnosed with influenza viral infections (Wang et al., 2011). It was, therefore, proposed that maxingshigan-yinqiaosan in combination with oseltamivir be utilized as an alternative medication in the treatment of influenza viral infections. In a multi-centric, randomized, double-blind, and placebo-controlled trial, a total of 480 adults with influenza symptoms were recruited. It was observed that TCM increased recovery by $17 \%(\mathrm{p}<0.001)$ and lowered disease severity (evaluated through the median symptom score) by $50 \%(\mathrm{p}<0.001)$ (Wang et al., 2010). Moreover, 136 influenza patients with wind-heat affecting Fei syndrome (WHAFS) were prescribed with Jinhua Qinggan Granules (JHG) that have been developed by experts in recent years. This preparation has been based on findings in a doubleblind randomized control trial. In this trial, the duration of defervescence, the defervescence rate, the efficacy of TCM, the alleviation rate of primary symptoms and physical signs of influenza, the absence of viral nucleic acid in pharyngeal secretions, and safety indices were assessed. It was shown that JHG was efficient and safe in treating patients with WHAFS (Li et al., 2013). Japanese doctors have conventionally prescribed TCM to treat influenza. This is based on findings of a randomized controlled trial that compared the efficacy of TCMs with NP inhibitors in treating influenza. It has been established that TCM was effectively tolerated when used to treat influenza. Meanwhile, TCM and NP inhibitors exhibited equivalent clinical efficacies against the influenza virus (Nabeshima et al., 2012). In the US, a randomized, double-blind placebo-controlled study established that Echinacea angustifolia DC, a compound used in herbal tea preparations was potent in alleviating influenza symptoms such as stuffiness, scratchy throat and fever (Lindenmuth and Lindenmuth, 2000). In agreement with our meta-analysis, RCTs 
demonstrated that TCM abbreviated fever time and showed a satisfactory impact in relieving cough and sore throat. In Table 1, we summarized the efficacy of TCMs in treating influenza (Table 1).

\section{Retrospective Analysis}

Besides meta-analysis RCT, researchers analyzed the duration of viral shedding in influenza patients admitted and administered with TCM in China. A total of 963 patients diagnosed with influenzavirus infection between May and July 2009 were recruited. The study showed that TCM therapy contributed to viral shedding among patients with a temperature of $\geq 38^{\circ} \mathrm{C}$ (Wang et al., 2012). Overall, these studies demonstrated that, besides alleviating symptoms of influenza patients, such as fever and cough, TCM have been proved to contribute to viral shedding.

\section{Use of TCM in Treating Influenza Among Children and During Pregnancy}

Treatment of influenza among children and pregnant women is challenging. However, other than effectively alleviating symptoms, TCM can compensate for deficiencies that cannot be bridged by conventional medicines in pregnant women. Children is the major group affected by influenza virus as they are usually vulnerable to complications. Moreover, the response of children to drug-drug metabolism is profoundly unique from that of adults. Therefore, it is essential to analyze the effects of TCM on children. It is, therefore, essential to analyze the effects of TCM on children. As mentioned above, ma-huang-tang was expansively used in China to manage children diagnosed with influenza. Ma-huang-tang has been widely used in China to manage influenza diagnoses among children. Without exhibiting adverse effects, it has been validated to be clinically useful in neonates, infants, and children with febrile viral symptoms (Nishimura et al., 2009). Additionally, it has been shown that it might potentially be useful in patients aged $\leq 5$ who have a low sensitivity to oseltamivir and experience problems when using zanamivir (Toriumi et al., 2012). Comparison of the efficacies of ma-huang-tang and oseltamivir was evaluated among children with type A influenza. Results showed that ma-huang-tang in combination with oseltamivir was capable of abbreviating fever duration when compared to the group administered with oseltamivir only (Kubo and Nishimura, 2007). Elsewhere, a systematic review focusing on the effects of Andrographis paniculata (burm.f.) nees on influenza infections among children reported that this herb minimized the duration of cough, sore throat and morbidity time compared to conventional medicines (Hu et al., 2017). The use of TCM aerosols in treating infantile influenza infections showed an effective rate of $99.03 \%$ with a cure rate of $65.38 \%$ (Ma et al., 2000). An investigation on the clinical efficacy of a sachet of Chinese herbs in preventing influenza among 239 children from Shanghai Baoshan Xubeihong Art Kindergarten revealed that the sachet alleviated symptoms such as fever, rhinocleisis, runny nose, and throat congestions. Equally, the incidence rates of influenza in the treatment group were low compared to the control group (Liu et al., 2010). Reports indicate that TCM is a valuable option during pregnancy. For instance, when influenza outbreaks occurred in the Middle East, the prevalence in the use of TCM varied between $22.3 \%$ 82.3\% in pregnant women (John and Shantakumari, 2015). Also, Across-sectional study conducted in the Central Appalachian Region showed that Trigonella foenum-graecum L. was regularly used in treating Influenza (Alwhaibi et al., 2017). More and more RCTs are focusing on the efficacy and safety of combination use of TCM and

TABLE 1 | Distribution of influenza article types and outcome indicators.

\begin{tabular}{|c|c|c|c|c|c|c|c|c|c|c|c|}
\hline \multirow[t]{3}{*}{ Study Design } & \multirow[t]{3}{*}{ Source } & \multirow[t]{3}{*}{ Groups } & \multirow[t]{3}{*}{$\mathbf{N}$} & \multicolumn{8}{|c|}{ Outcomes } \\
\hline & & & & \multicolumn{6}{|c|}{ Main Outcomes } & \multicolumn{2}{|c|}{ Secondary Outcomes } \\
\hline & & & & Fever & Headache & Myalgia & Cough & Sorethroat & Malaise & Hospitalization & $\begin{array}{c}\text { Side } \\
\text { effects }\end{array}$ \\
\hline \multirow[t]{2}{*}{ Meta } & $\begin{array}{l}\text { Li J. H. et al., } \\
2016\end{array}$ & $\begin{array}{l}\text { 1.Ma Huang Tang plus } \\
\text { NAls vs. NAls } \\
\text { 2. Ma Huang Tang vs. } \\
\text { NAls }\end{array}$ & $\begin{array}{l}30 \text { studies, } \\
3,444 \text { cases }\end{array}$ & $\uparrow$ & $\uparrow$ & $\uparrow$ & & & $\uparrow$ & $\uparrow$ & $\uparrow$ \\
\hline & $\begin{array}{l}\text { Yoshino et } \\
\text { al., } 2019 \\
\text { Hu et al., } \\
2017\end{array}$ & $\begin{array}{l}\text { 1.TCM VS oseltamivir } \\
2 . \mathrm{TCM}+\text { oseltamivir } \\
\text { vs. oseltamivir } \\
\text { Andrographis } \\
\text { paniculata vs. placebo }\end{array}$ & $\begin{array}{l}12 \text { studies, } \\
1,170 \text { cases } \\
33 \text { studies, } \\
7,175 \text { cases }\end{array}$ & $\uparrow$ & & & $\uparrow$ & $\uparrow$ & & $\uparrow$ & \\
\hline \multirow[t]{5}{*}{$\begin{array}{l}\text { Randomized } \\
\text { Controlled Trial }\end{array}$} & $\begin{array}{l}\text { Wang et al., } \\
2011\end{array}$ & $\begin{array}{l}\text { maxingshigan- } \\
\text { yinqiaosan vs. } \\
\text { Oseltamivir }\end{array}$ & 410 cases & $\uparrow$ & $\uparrow$ & & $\uparrow$ & $\uparrow$ & & & \\
\hline & $\begin{array}{l}\text { Wang et al., } \\
2010\end{array}$ & Antiwei vs. placebo & 480 cases & $\uparrow$ & $\uparrow$ & $\uparrow$ & $\uparrow$ & $\uparrow$ & & & \\
\hline & $\begin{array}{l}\text { Li et al., } \\
2013\end{array}$ & $\begin{array}{l}\text { Echinacea vs. } \\
\text { Placebo }\end{array}$ & 136 cases & & & & & & & & \\
\hline & $\begin{array}{l}\text { Nabeshima } \\
\text { et al., } 2012\end{array}$ & $\begin{array}{l}\text { Jinhua Qinggan } \\
\text { Granules vs. Placebo }\end{array}$ & 28 cases & $\uparrow$ & $\uparrow$ & $\uparrow$ & $\uparrow$ & $\uparrow$ & & & $\uparrow$ \\
\hline & $\begin{array}{l}\text { Lindenmuth } \\
\text { et al., } 2013\end{array}$ & $\begin{array}{l}\text { Maoto VS oseltamivir } \\
\text { vs. zanamivir }\end{array}$ & 95 cases & $\uparrow$ & $\uparrow$ & $\uparrow$ & $\uparrow$ & $\uparrow$ & & & $\uparrow$ \\
\hline
\end{tabular}

The upward arrow represents the relief of symptoms after treatment. 
conventional therapy (He et al., 2019). In summary, there is potential value in using TCM to treat influenza among children and pregnant women; nevertheless, more investigations are necessary for clinical validations.

\section{BASIC EXPERIMENTS}

\section{Direct Anti-Influenza Virus by Interfering the Invasion Process}

In the clinical set-up, we have witnessed the effects of TCM in improving the symptoms of influenza and cutting the course of the disease. However, how TCM act on influenza virus and its effects on the host requires a comprehensive analysis using basic experiments. Recently, researchers have constantly been drawn to investigate the therapeutic effects of TCM. Several types of TCM including, coptis deltoidea (C.Y.Cheng \& P.K.Hsiao), Isatis tinctoria L., Lonicera japonica thunb., scutellaria baicalensis georgi., cyrtomium fortunei J.Sm., Houttuynia cordata thunb., gardenia jasminoides J.Ellis, and chrysanthemum indicum L., and other TCM prescriptions have been proven to effectively suppress influenza virus (Han et al., 2016).

\section{Single Herbal Medicine}

The influenza virus invades the human body in six steps, that is, adsorption, penetration, shelling, biosynthesis, assembly, and release. As its mode of action, TCM targets these six steps. i) The extracts from single herbal medicine: Lonicera japonica thunb. HSencoded atypical microRNA was verified to inhibit influenza viral replication in vitro and in vivo. Furthermore, HS decoction significantly minimized mice mortalities from influenza virus infection (Zhou et al., 2015). Extracts of Laggera crispata (vahl) (Hepper \& J.R.I.Wood), a TCM have been shown to inhibit NA activity. It has also been reported that this compound could suppress NF- $\kappa \mathrm{B}$ signaling pathways and viral RNP complexes from the nucleus thereby inhibiting viral replication (Guan et al., 2017). Besides blocking viral replication, TCM enhances the prognosis of mice infected by influenza. Yan et al. evaluated the effects of berberine, a natural isoquinoline alkaloid isolated from coptis chinensis franch. on influenza virus. Their results showed that berberine suppressed viral replication in A549 cells and the lungs of mice. Compared to conventional medicines, this compound exhibited significant anti-inflammatory effects on the pulmonary system and reduced necrosis in mice. It was also observed that inflammatory cell infiltrations and pulmonary edemas due to viral infections in mice were reduced (Yan Y. Q. et al., 2018). Houttuynia cordata Thunb (HCP), a TCM, plays a pivotal role in the treatment of bacterial and viral respiratory infections. Accompanied by a reduction in virus replication, HCP is reported to increase the overall survival rate of mice infected with influenza. Further studies have proved that it might be associated with the decrease in the concentration of pulmonary proinflammatory cytokines/ chemokines and the number of intestinal goblet cells, which resulting in upregulation of sIgA and tight junction protein ( $\mathrm{ZO}-$ 1) in the intestine (Zhu et al., 2018). Researchers at the Kitasato Institute in Japan extracted flavonoids from the roots and leaves of
Astragalus mongholicus bunge and confirmed their capacity in preventing the growth of influenza virus. It has been reported that glycyrrhizic acid significantly inhibits influenza virus (Pompei et al., 1983; Baltina et al., 2009). Besides, extracts of Paeonia delavayi Franch. and pogostemon cablin (blanco) benth. were found to efficiently inhibit NP (Li J. et al., 2016; Liu et al., 2016). The roots of Bupleurum marginatum var. stenophyllum (H.Wolff) had inhibitory effects on the replication of influenza (Fang et al., 2017). Importantly, it has been confirmed that Tetradium ruticarpum (A.Juss.) T.G.Hartley effectively hindered viral attachment and penetration into host cells (Lin et al., 2016). ii) Single components: A study by Li et al. addressed that, Dendrobine, extracted from a traditional Chinese herb (dendrobium nobile lindl.), interfered with early viral replication and bound to the viral NP thereby restricting nuclear export of viral NP and its oligomerization (Li et al., 2017). HESA-A is a natural ingredient retrieved from marine herbs. This compound blocks viral penetration into the cell (Mehrbod et al., 2014). Persicaria chinensis (l.) h. gross, from Vietnam, was shown to target NP, including oseltamivir-resistant ones (Tran et al., 2017). These results show that single herbal medicines inhibit viral proliferation by targeting multiple sites and pathways during replication. These results are presented in Figure 1.

\section{Traditional Chinese Herbal Prescription}

Besides the single Chinese herbal medicine, traditional Chinese herbal prescription has precariously shaped the academic debate on influenza disease management. Huang-Lian-Jie-Du-Tang (composed of coptis chinensis Franch., scutellaria baicalensis georgi, phellodendron chinense C.K.Schneid., and gardenia jasminoides J.Ellis at the weight ratio of $3: 2: 2: 3$ ) (HLJDT) is a classical prescription composed of 4 TCM herbs that have been used to treat diverse diseases including influenza. In total, 13 compounds have been isolated from the plasma profile of HLJDT. These compounds include, jatrorrhizine, palmatine, epiberberine, geniposide, oroxylin A, berberine, coptisine, baicalein, wogonoside, phellodendrine, wogonin, oroxylin A-7O-glucuronide, and baicalin. They have been shown to be potent NA-1 inhibitors (Zhou et al., 2017). Xin-Jia-Xiang-Ru-Yin [(composed of mosla chinensis maxim., lonicera japonica thunb., dolichos lablab L., magnolia officinalis rehd. et wils. and forsythia suspensa (thunb.) vahl at the weight ratio of 2:3:3:2:3)], a traditional Chinese prescription used for managing summer influenza in China, has been demonstrated to integrate antiviral therapy and immune modulation effects by lowering the expression of Interferon- $\gamma($ IFN- $\gamma)$ and Signal transducer and activator of transcription-1 (STAT1), resulting to reduced inflammation hence harboring key therapeutic benefits against summer influenza (Li et al., 2018). The Re Du Ping intravenous injection, a TCM can adsorb the virus into host cells and inhibit their proliferation. This injection also has a direct viricidal effect (Jing et al., 2010). Studies into the pharmacodynamics and molecular mechanisms of Qingqi Liangying granules have reported that they participate in Wnt signaling pathways by regulating virus invasions on epithelial cell signaling pathway, thereby, inhibiting viral replication (Wang et al., 2017). Chinese 


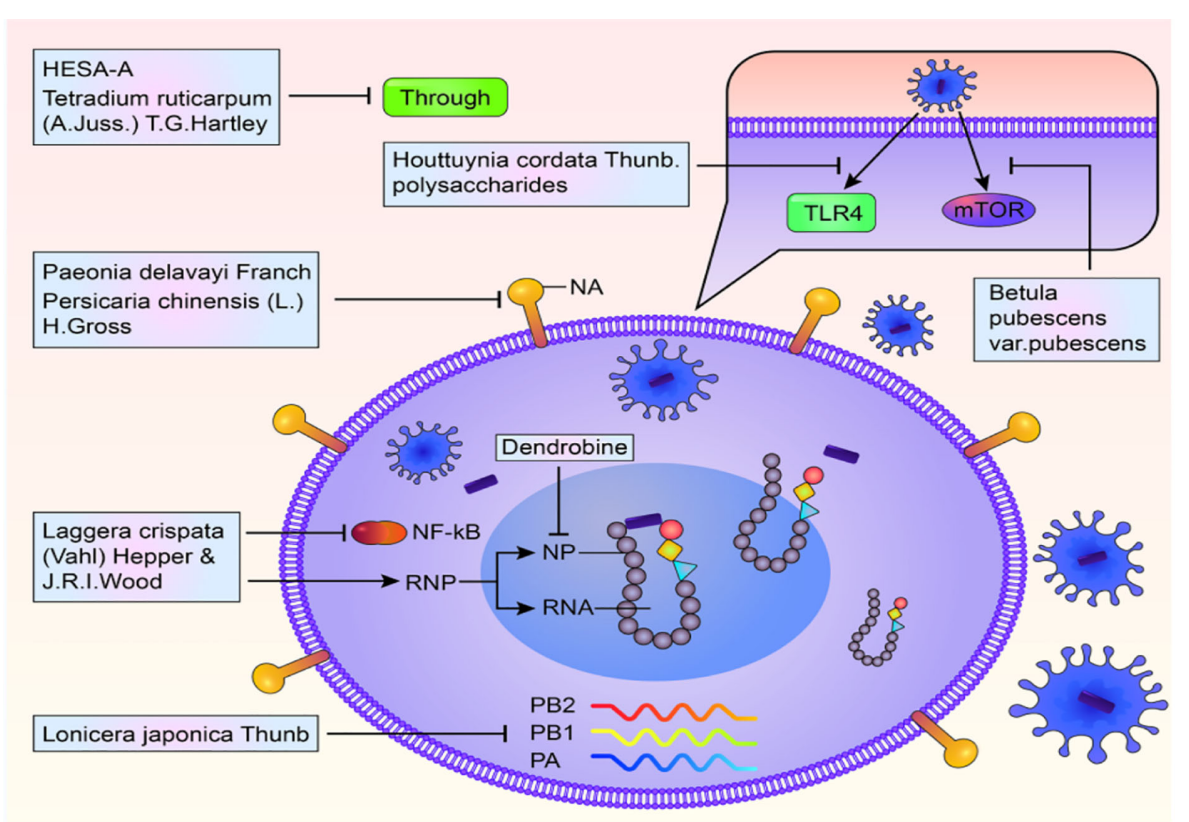

FIGURE 1 | Multiple targets and multiple pathways to inhibit influenza virus.

classical herbal prescription was also used in the prevention of influenza. It was reported to reduce the vulnerability of cells to the invasion of influenza virus and alleviate viral induced lung lesions (Liu et al., 2013).

\section{In-Direct Anti-Influenza Virus by Regulating Host Immune System}

In addition to the direct effect on the influenza virus, TCM regulates immune activation and signaling pathways that impact on the influenza virus. The host innate immune response to influenza virus occurs as follows: Infection with influenza virus stimulates the body's innate immune response, which involves multiple effector cells, immune molecules, and factors, such as Interleukin-6 (IL-6) and Interferon- $\alpha$ (IFN- $\alpha$ ), to effectively regulate viral replication (Hayden et al., 1998). Several experiments have confirmed that TCM is the most potent antiinflammatory natural medicine which can modulate all kinds of cytokines and immune molecules.

\section{Traditional Chinese Herbal Prescription}

A study conducted on the mechanisms of TCM prescription in the treatment of influenza deduced that anti-inflammatory effects conferred by TCM protect against inflammatory injuries induced by the influenza virus in mice. These herbal medicines significantly reduce the expression of proinflammatory cytokines Tumor Necrosis Factor- $\alpha$ (TNF- $\alpha$ ) and IL-6 in lung tissues of infected mice (Wang et al., 2005; $\mathrm{Xu}$ et al., 2014). Sheng Jiang San (SJS), (rheum officinale baill., bombyx batryticatus, cicadae periostracum, and curcuma longa $L$. in a ratio of $4: 2: 1: 3)$, a traditional multi-herb prescription, used to treat influenza infections. Research has shown that SJS down-regulates TNF- $\alpha$ and up-regulates IL-2 in influenza infected mice. Equally, lung indices used to evaluate the level of inflammation and viral loads in SJS treated mice were markedly decreased compared to the controls (Zhang et al., 2018). Currently, Yinhuapinggan granule (YHPG), a Chinese drug with patent that originated from ma-huang-tang is used in the treatment of influenza. A study conducted by Du revealed that YHPG markedly inhibited replication of the influenza virus and significantly up-regulated the levels of IFN- $\beta$ and IFN-stimulated genes (Mx-1, ISG-15, and ISG-56) compared to the control group (Du et al., 2018). The Yinqiao powder [15 g of forsythia suspensa (thunb.) vahl, $15 \mathrm{~g}$ of lonicera confusa DC., $9 \mathrm{~g}$ of platycodon grandiflorus (jacq.) A.DC., $9 \mathrm{~g}$ of mentha canadensis L., $6 \mathrm{~g}$ of lophatherum gracile brongn., $5 \mathrm{~g}$ of glycyrrhiza uralensis fisch. ex DC., $6 \mathrm{~g}$ of nepeta tenuifolia benth., $6 \mathrm{~g}$ of glycine max (L.) merr., $6 \mathrm{~g}$ of arctium lappa L., $10 \mathrm{~g}$ of phragmites australis subsp. australis]; The Xinjiaxiangruyin concoction [6 g of mosla chinensis maxim., $9 \mathrm{~g}$ of lonicera confusa DC., $9 \mathrm{~g}$ of lablab purpureus subsp. purpureus, $6 \mathrm{~g}$ of magnolia officinalis rehder \& E.H.Wilson, $6 \mathrm{~g}$ of forsythia suspensa (thunb.) vahl]; and the Guizhi-andMahuang decoction (9 $\mathrm{g}$ of ephedra sinica stapf, $6 \mathrm{~g}$ of cinnamomum cassia (L.) J.Presl, $9 \mathrm{~g}$ of prunus armeniaca L., $6 \mathrm{~g}$ of glycyrrhiza uralensis fisch. ex DC., $9 \mathrm{~g}$ of paeonia lactiflora pall., $9 \mathrm{~g}$ of zingiber officinale roscoe, $3 \mathrm{~g}$ of ziziphus jujuba mill.] 
are widely applied in clinical influenza treatment. These compounds reduce the expression levels of TLR7, MyD88, IRAK4, and NF- $\mathrm{BB}$ thereby by regulating the Toll-Like Receptor7/Nuclear Factor Kappa-B (TLR7/NF-אB) signaling pathway (Fu et al., 2018). Yi-Zhi-Hao pellet (CYZH) is a popular Chinese prescription used in influenza treatment. Mechanistically, CYZH lacked an inhibitory effect on viral protein HA and IAV RNA-dependent RNA polymerase but inhibited IAV replication by activating the Nrf2/HO-1 pathway (Yin et al., 2017). Lianhuaqingwen, a TCM prescription alleviates viral-induced gene expressions of IL-6, IL-8, TNF-a, IP-10, and MCP-1 (Ding et al., 2017). Furthermore, Lianhuaqingwen treatment resulted in abnormal particle morphology of virion in cells (Runfeng et al., 2020). San Wu Huangqin (SWHD) decoction (sophora flavescens, scutellaria baicalensis, and rehmannia glutinosa at a ratio of 1:1:2) exhibits its anti-viral effects by regulating the immune system (Ma et al., 2018). The Ge Gen Decoction [12 g of pueraria montana var. lobata (Willd.) maesen \& S.M.Almeida ex sanjappa \& predeep, $9 \mathrm{~g}$ of ephedra sinica stapf, $6 \mathrm{~g}$ of cinnamomum cassia (L.) J.Presl, $6 \mathrm{~g}$ of glycyrrhiza uralensis fisch. ex DC., $6 \mathrm{~g}$ of paeonia lactiflora pall., $9 \mathrm{~g}$ of zingiber officinale roscoe, and $22 \mathrm{~g}$ of ziziphus jujuba mill.] decreased the expression of TNF- $\alpha$ and toll-like receptor 7 signaling pathways in influenza virus infected mice thereby reducing lung inflammation (Geng et al., 2019). Research endorsed that Gui Zhi Ma Huang Ge Ban Tang $[9$ g of ephedra sinica stapf, $6 \mathrm{~g}$ of cinnamomum cassia (L.) J.Presl, $9 \mathrm{~g}$ of prunus amygdalus batsch, and $6 \mathrm{~g}$ of glycyrrhiza uralensis fisch. ex DC., $9 \mathrm{~g}$ of paeonia lactiflora pall., $9 \mathrm{~g}$ of zingiber officinale roscoe, and $10 \mathrm{~g}$ of ziziphus jujuba mill.] exhibit a good ability to reduce the proportion of Helper $\mathrm{T}$ cell 1/Helper $\mathrm{T}$ cell 2(Th1/Th2) and Helper T cell 1/Regulatory cells (Th17/Treg) cells thus reducing lung inflammation (Qin et al., 2018). Artemisia scoparia Waldst. $\&$ Kit. is expansively distributed in Xinjiang China and reported to downregulate NF- $\mathrm{KB}$ signal pathway thereby inhibiting viral replication (Yan H. et al., 2018). Summarily, TCM regulates signaling pathways like NF- $\mathrm{BB}$ and inflammatory factors such as TNF- $\alpha$ and IL-6, that are important in modulating immune responses against the virus.

\section{Single Herbal Medicine}

Besides the TCM prescription, single herbal medicine indirectly plays a crucial role in anti-viral activity. i) The extracts from single herbal medicine: As mentioned above, laggera pterodonta, demonstrated a wide spectrum of anti-influenza virus activity via the NF- $\kappa \mathrm{B}$ pathway, COX-2, and $\mathrm{p} 38 / \mathrm{MAPK}$ pathway (Wang et al., 2017). Moreover, flavonoids extracted from Scutellaria baicalensis Georgi (FESR) could inhibit excessive activations of the complement system, thereby, improving acute lung injuries (Zhi et al., 2020). Houttuynia cordata thunb polysaccharide (HCP) aids systemic influenza treatment by locally acting on the intestines and balancing the microbiota (Chen et al., 2019). Bupleurum falcatum L., extracted from a traditional Chinese herb, was also found to be an immune modulator (Yao et al., 2018). Isatis tinctoria L., attenuates viral-induced NF- $\kappa \mathrm{B}$ activation (Li et al., 2015). Research has shown that Forsythia suspensa (thunb.) vahl interferes with the budding process of newly formed virions and reduces influenza M1 protein that is necessary for viral spread (Law et al., 2017). ii) Single components: Baicalin, a popularly known herbal medicine ingredient in China for treating Fei-Re syndrome characterized by fever, and cough, can be extracted from scutellaria baicalensis georgi, which was reported to induce autophagy so as to inhibit mTOR signaling pathway. Thus, shedding more light on the development of novel anti-influenza drugs (Zhu et al., 2015). Quercetin-7-O-glucoside was discovered in many types of Chinese herb and demonstrated a strong inhibition against influenza $\mathrm{A}$ and $\mathrm{B}$ viruses by downsizing virus-induced ROS and autophagy formation (Gansukh et al., 2016). The polysaccharides extracted from the roots of astragalus mongholicus Bunge species, a famous TCM used for hundreds of years to improve QI with Chinese medicine theory, was ratified to modulate Th1/Th2 balance and secretions of IFN- $\gamma$, IL-17A, and IgG2a (Yakuboğulları et al., 2019). These studies testify that different types of TCMs possess remarkable potential in immunomodulation against influenza virus.

\section{SUMMARY}

In conclusion, TCM impacts on the prevention and treatment of influenza. It has a potential value in shorting fever durations and alleviating influenza symptoms among children and pregnant women. However, the side effects of TCM in children and pregnant women are still elusive, which needs more clinical trials about the safety and vivo toxicity. These medicines also regulate the immune system. Their modes of action involve inhibiting NA, viral replication, and stopping viral entry into the cell. The synergistic effects of TCM and conventional medicines are encouraging as an avenue for influenza therapy.

\section{AUTHOR CONTRIBUTIONS}

YX: Conceptualization, Writing-original draft. ND: Conceptualization. XL: Writing-original draft. BL: Writingoriginal draft. HZ: Writing-original draft. CZ: Supervision. LL: Supervision. CL: Supervision. LH: Funding acquisition and supervision.

\section{ACKNOWLEDGMENTS}

This work was supported by the National Science and Technology Major Project (2018ZX10101001-005) and by the China Academy of Chinese Medical Sciences Project (Nos. Z0656 and Z0606). The authors have no conflicts of interest to declare. 


\section{REFERENCES}

Alwhaibi, M., Goyat, R., and Kelly, K. M. (2017). The Use of Herbal Remedies among Mothers of Young Children Living in the Central Appalachian Region. Evid. Based. Complement. Alternat. Med. 2017:1739740. doi: 10.1155/2017/ 1739740

Baltina, L. A., Kondratenko, R. M., Baltina, L. A.Jr., Plyasunova, O. A., Pokrovskii, A. G., and Tolstikov, G. A. (2009). Prospects for the creation of new antiviral drugs based on glycyrrhizic acid and its derivatives (a review). Pharm. Chem. J. 43 (10), 539-548. doi: 10.1007/s11094-010-0348-2

Birnkrant, D., and Cox, E. (2009). The Emergency Use Authorization of peramivir for treatment of 2009 H1N1 influenza. N. Engl. J. Med. 361 (23), 2204-2207. doi: 10.1056/NEJMp0910479

Brody, H. (2019). Influenza. Nature 573 (7774), S49. doi: 10.1038/d41586-01902750-x

Chang, Q., Wo, S., Ngai, K. L., Wang, X., Fok, B., Ngan, T. M., et al. (2014). Bench to bed evidences for pharmacokinetic and pharmacodynamic interactions involving oseltamivir and chinese medicine. Evid. Based. Complement. Alternat. Med. 2014:354172. doi: 10.1155/2014/354172

Chen, M. Y., Li, H., Lu, X. X., Ling, L. J., Weng, H. B., Sun, W., et al. (2019). Houttuynia cordata polysaccharide alleviated intestinal injury and modulated intestinal microbiota in H1N1 virus infected mice. Chin. J. Nat. Med. 17 (3), 187-197. doi: 10.1016/s1875-5364(19)30021-4

Cox, N. J., and Subbarao, K. (2000). Global epidemiology of influenza: past and present. Annu. Rev. Med. 51, 407-421. doi: 10.1146/annurev.med.51.1.407

Ding, Y., Dou, J., Teng, Z., Yu, J., Wang, T., Lu, N., et al. (2014). Antiviral activity of baicalin against influenza A (H1N1/H3N2) virus in cell culture and in mice and its inhibition of neuraminidase. Arch. Virol. 159 (12), 3269-3278. doi: 10.1007/s00705-014-2192-2

Ding, Y., Zeng, L., Li, R., Chen, Q., Zhou, B., Chen, Q., et al. (2017). The Chinese prescription lianhuaqingwen capsule exerts anti-influenza activity through the inhibition of viral propagation and impacts immune function. $B M C$ Complement. Altern. Med. 17 (1), 130. doi: 10.1186/s12906-017-1585-7

Du, H. X., Zhou, H. F., Wan, H. F., Yang, J. H., Lu, Y. Y., He, Y., et al. (2018). Antiviral effects and mechanisms of Yinhuapinggan granule against H1N1 influenza virus infection in RAW264.7 cells. Inflammopharmacology 26 (6), 1455-1467. doi: 10.1007/s10787-018-0457-1

Fang, W., Yang, Y. J., Guo, B. L., and Cen, S. (2017). Anti-influenza triterpenoid saponins (saikosaponins) from the roots of Bupleurum marginatum var. stenophyllum. Bioorg. Med. Chem. Lett. 27 (8), 1654-1659. doi: 10.1016/ j.bmcl.2017.03.015

Fu, Y. J., Yan, Y. Q., Qin, H. Q., Wu, S., Shi, S. S., Zheng, X., et al. (2018). Effects of different principles of Traditional Chinese Medicine treatment on TLR7/NFКB signaling pathway in influenza virus infected mice. Chin. Med. 13, 42. doi: 10.1186/s13020-018-0199-4

Gaitonde, D. Y., Moore, F. C., and Morgan, M. K. (2019). Influenza: Diagnosis and Treatment. Am. Fam. Physician 100 (12), 751-758.

Gansukh, E., Kazibwe, Z., Pandurangan, M., Judy, G., and Kim, D. H. (2016). Probing the impact of quercetin-7-O-glucoside on influenza virus replication influence. Phytomedicine 23 (9), 958-967. doi: 10.1016/j.phymed.2016.06.001

Geng, Z. K., Li, Y. Q., Cui, Q. H., Du, R. K., and Tian, J. Z. (2019). Exploration of the mechanisms of Ge Gen Decoction against influenza A virus infection. Chin. J. Nat. Med. 17 (9), 650-662. doi: 10.1016/s1875-5364(19)30079-2

Govorkova, E. A., Leneva, I. A., Goloubeva, O. G., Bush, K., and Webster, R. G. (2001). Comparison of efficacies of RWJ-270201, zanamivir, and oseltamivir against H5N1, H9N2, and other avian influenza viruses. Antimicrob. Agents Chemother. 45 (10), 2723-2732. doi: 10.1128/aac.45.10.2723-2732.2001

Guan, W., Li, J., Chen, Q., Jiang, Z., Zhang, R., Wang, X., et al. (2017). Pterodontic Acid Isolated from Laggera pterodonta Inhibits Viral Replication and Inflammation Induced by Influenza A Virus. Molecules 22 (10), 1738. doi: 10.3390/molecules 22101738

Han, X., Zhang, D. K., Guo, Y. M., Feng, W. W., Dong, Q., Zhang, C. E., et al. (2016). Screening and evaluation of commonly-used anti-influenza Chinese herbal medicines based on anti-neuraminidase activity. Chin. J. Nat. Med. 14 (10), 794-800. doi: 10.1016/s1875-5364(16)30095-4

Hayden, F. G., Fritz, R., Lobo, M. C., Alvord, W., Strober, W., and Straus, S. E. (1998). Local and systemic cytokine responses during experimental human influenza A virus infection. Relation to symptom formation and host defense. J. Clin. Invest. 101 (3), 643-649. doi: 10.1172/jci1355

He, W., Han, H., Wang, W., and Gao, B. (2011). Anti-influenza virus effect of aqueous extracts from dandelion. Virol. J. 8:538. doi: 10.1186/1743-422x-8-538

He, J., Li, Z., Huang, W., Guan, W., Ma, H., Yang, Z. F., et al. (2019). Efficacy and safety of Chou-Ling-Dan granules in the treatment of seasonal influenza via combining Western and traditional Chinese medicine: protocol for a multicentre, randomised controlled clinical trial. BMJ Open 9 (4), e024800. doi: 10.1136/bmjopen-2018-024800

Hu, X. Y., Wu, R. H., Logue, M., Blondel, C., Lai, L. Y. W., Stuart, B., et al. (2017). Andrographis paniculata (Chuān Xīn Lián) for symptomatic relief of acute respiratory tract infections in adults and children: A systematic review and meta-analysis. PloS One 12 (8), e0181780. doi: 10.1371/journal.pone.0181780

Huang, I. C., Li, W., Sui, J., Marasco, W., Choe, H., and Farzan, M. (2008). Influenza A virus neuraminidase limits viral superinfection. J. Virol. 82 (10), 4834-4843. doi: 10.1128/jvi.00079-08

Jing, S., Gu, L. G., and Zhou, Y. (2010). [Effect of dureping injection on T-cells function and their function in killing FM1 infected Mphi in vitro]. Zhongguo Zhong Xi Yi Jie He Za Zhi 30 (7), 729-732.

JJ, X. (2010). Traditional Chinese Medicine Treatment of Viral Infectious Diseases Vol. 2010 (Beijing: Science and Technology Press).

John, L. J., and Shantakumari, N. (2015). Herbal Medicines Use During Pregnancy: A Review from the Middle East. Oman Med. J. 30 (4), 229-236. doi: $10.5001 /$ omj.2015.48

Kamali, A., and Holodniy, M. (2013). Influenza treatment and prophylaxis with neuraminidase inhibitors: a review. Infect. Drug Resist. 6, 187-198. doi: 10.2147/ idr.S36601

Kawaoka, Y. (2006). Influenza Virology: Current Topics (England: Caister Academic Press).

Kiso, M., Mitamura, K., Sakai-Tagawa, Y., Shiraishi, K., Kawakami, C., Kimura, K., et al. (2004). Resistant influenza A viruses in children treated with oseltamivir: descriptive study. Lancet 364 (9436), 759-765. doi: 10.1016/s0140-6736(04) 16934-1

Kubo, T., and Nishimura, H. (2007). Antipyretic effect of Mao-to, a Japanese herbal medicine, for treatment of type A influenza infection in children. Phytomedicine 14 (2-3), 96-101. doi: 10.1016/j.phymed.2006.09.015

Law, A. H., Yang, C. L., Lau, A. S., and Chan, G. C. (2017). Antiviral effect of forsythoside A from Forsythia suspensa (Thunb.) Vahl fruit against influenza A virus through reduction of viral M1 protein. J. Ethnopharmacol. 209, 236247. doi: 10.1016/j.jep.2017.07.015

Lee, J., Park, J. H., Jwa, H., and Kim, Y. H. (2017). Comparison of Efficacy of Intravenous Peramivir and Oral Oseltamivir for the Treatment of Influenza: Systematic Review and Meta-Analysis. Yonsei Med. J. 58 (4), 778-785. doi: $10.3349 / y m j .2017 .58 .4 .778$

Li, G. Q., Zhao, J., Tu, Z. T., Li, J. B., Liu, Q. Q., Shi, L. Q., et al. (2013). [Treating influenza patients of wind-heat affecting Fei syndrome by jinhua qinggan granule: a double-blinded randomized control trial]. Zhongguo Zhong Xi Yi Jie He Za Zhi 33 (12), 1631-1635.

Li, C., Fang, J. S., Lian, W. W., Pang, X. C., Liu, A. L., and Du, G. H. (2015). In vitro antiviral effects and 3D QSAR study of resveratrol derivatives as potent inhibitors of influenza H1N1 neuraminidase. Chem. Biol. Drug Des. 85 (4), 427-438. doi: 10.1111/cbdd.12425

Li, J., Zhou, B., Li, C., Chen, Q., Wang, Y., Li, Z., et al. (2015). Lariciresinol-4-O- $\beta$ D-glucopyranoside from the root of Isatis indigotica inhibits influenza A virusinduced pro-inflammatory response. J. Ethnopharmacol. 174, 379-386. doi: 10.1016/j.jep.2015.08.037

Li, J. H., Wang, R. Q., Guo, W. J., and Li, J. S. (2016). Efficacy and safety of traditional Chinese medicine for the treatment of influenza A (H1N1): A metaanalysis. J. Chin. Med. Assoc. 79 (5), 281-291. doi: 10.1016/j.jcma.2015.10.009

Li, J., Yang, X., and Huang, L. (2016). Anti-Influenza Virus Activity and Constituents. Characterization of Paeonia delavayi Extracts. Molecules 21 (9), 1133. doi: $10.3390 /$ molecules 21091133

Li, R., Liu, T., Liu, M., Chen, F., Liu, S., and Yang, J. (2017). Anti-influenza A Virus Activity of Dendrobine and Its Mechanism of Action. J. Agric. Food Chem. 65 (18), 3665-3674. doi: 10.1021/acs.jafc.7b00276

Li, Q., Pang, P., Zheng, K., Sun, L., Wang, J., and Chen, X. (2018). Xin-Jia-XiangRu-Yin alleviated H1N1-induced acute lung injury and inhibited the IFN- $\gamma$ - 
related regulatory pathway in summer flu. BioMed. Pharmacother. 108, 201207. doi: 10.1016/j.biopha.2018.09.022

Lin, T. J., Lin, C. F., Chiu, C. H., Lee, M. C., and Horng, J. T. (2016). Inhibition of endosomal fusion activity of influenza virus by Rheum tanguticum (da-huang). Sci. Rep. 6:27768. doi: 10.1038/srep27768

Lindenmuth, G. F., and Lindenmuth, E. B. (2000). The efficacy of echinacea compound herbal tea preparation on the severity and duration of upper respiratory and flu symptoms: a randomized, double-blind placebocontrolled study. J. Altern. Complement. Med. 6 (4), 327-334. doi: 10.1089/ 10755530050120691

Liu, L., Yue, X. Q., Wang, L. N., Gu, W., Xin, H. L., Zheng, G. Y., et al. (2010). [Preventive effects of a sachet of Chinese herbs on influenza and its immune regulation]. Zhong Xi Yi Jie He Xue Bao 8 (10), 949-954. doi: 10.3736/ jcim20101006

Liu, Q., Lu, L., Hua, M., Xu, Y., Xiong, H., Hou, W., et al. (2013). JiaweiYupingfeng-Tang, a Chinese herbal formula, inhibits respiratory viral infections in vitro and in vivo. J. Ethnopharmacol. 150 (2), 521-528. doi: 10.1016/j.jep.2013.08.056

Liu, F., Cao, W., Deng, C., Wu, Z., Zeng, G., and Zhou, Y. (2016). Polyphenolic glycosides isolated from Pogostemon cablin (Blanco) Benth. as novel influenza neuraminidase inhibitors. Chem. Cent J. 10, 51. doi: 10.1186/s13065-016-0192-x

Lu, Z. Y. (2008). ZN. Internal Medicine (Beijing: People's Medical Publishing House).

Ma, B., Duan, X., and Wang, Z. (2000). [Clinical and experimental study on Shuanghua aerosol in treating infantile upper respiratory tract infection]. Zhongguo Zhong Xi Yi Jie He Za Zhi 20 (9), 653-655.

Ma, Q., Yu, Q., Xing, X., Liu, S., Shi, C., and Luo, J. (2018). San Wu Huangqin Decoction, a Chinese Herbal Formula, Inhibits Influenza a/PR/8/34 (H1N1) Virus Infection In Vitro and In Vivo. Viruses 10 (3), 117. doi: 10.3390/ v10030117

Mehrbod, P., Ideris, A., Omar, A. R., and Hair-Bejo, M. (2014). Prophylactic effect of herbal-marine compound (HESA-A) on influenza A virus infectivity. BMC Complement. Altern. Med. 14:131. doi: 10.1186/1472-6882-14-131

Moscona, A. (2004). Oseltamivir-resistant influenza? Lancet 364 (9436), 733-734. doi: 10.1016/s0140-6736(04)16947-x

Moscona, A. (2009). Global transmission of oseltamivir-resistant influenza. N. Engl. J. Med. 360 (10), 953-956. doi: 10.1056/NEJMp0900648

Nabeshima, S., Kashiwagi, K., Ajisaka, K., Masui, S., Takeoka, H., Ikematsu, H., et al. (2012). A randomized, controlled trial comparing traditional herbal medicine and neuraminidase inhibitors in the treatment of seasonal influenza. J. Infect. Chemother. 18 (4), 534-543. doi: 10.1007/s10156-012-0378-7

Nagai, E., Iwai, M., Koketsu, R., Okuno, Y., Suzuki, Y., Morimoto, R., et al. (2019). Anti-Influenza Virus Activity of Adlay Tea Components. Plant Foods Hum. Nutr. 74 (4), 538-543. doi: 10.1007/s11130-019-00773-3

Nishimura, N., Doi, N., Uemura, T., Taketani, T., Hayashi, G., Kasai, T., et al. (2009). [Pharmaceutical analysis and clinical efficacy of Kampo medicine, maoto, extract suppository against pediatric febrile symptoms]. Yakugaku Zasshi 129 (6), 759-766. doi: 10.1248/yakushi.129.759

Panning, M. (2013). [Influenza today and in the future]. Pneumol. (Berl) 10 (5), 314-325. doi: 10.1007/s10405-013-0674-7

Pompei, R., Paghi, L., Ingianni, A., and Uccheddu, P. (1983). Glycyrrhizic acid inhibits influenza virus growth in embryonated eggs. Microbiologica 6 (3), 247250.

Qin, H. Q., Shi, S. S., Fu, Y. J., Yan, Y. Q., Wu, S., Tang, X. L., et al. (2018). Effects of Gui Zhi Ma Huang Ge Ban Tang on the TLR7 Pathway in Influenza Virus Infected Mouse Lungs in a Cold Environment. Evid. Based. Complement. Alternat. Med. 2018:5939720. doi: 10.1155/2018/5939720

Rajasekaran, D., Palombo, E. A., Chia Yeo, T., Lim Siok Ley, D., Lee Tu, C., Malherbe, F., et al. (2013). Identification of traditional medicinal plant extracts with novel anti-influenza activity. PloS One 8 (11), e79293. doi: 10.1371/ journal.pone.0079293

Runfeng, L., Yunlong, H., Jicheng, H., Weiqi, P., Qinhai, M., Yongxia, S., et al. (2020). Lianhuaqingwen exerts anti-viral and anti-inflammatory activity against novel coronavirus (SARS-CoV-2). Pharmacol. Res. 156:104761. doi: 10.1016/j.phrs.2020.104761

Shetty, A. K., and Peek, L. A. (2012). Peramivir for the treatment of influenza. Expert Rev. Anti Infect. Ther. 10 (2), 123-143. doi: 10.1586/eri.11.174

Tian, L., Wang, Z., Wu, H., Wang, S., Wang, Y., Wang, Y., et al. (2011). Evaluation of the anti-neuraminidase activity of the traditional Chinese medicines and determination of the anti-influenza A virus effects of the neuraminidase inhibitory TCMs in vitro and in vivo. J. Ethnopharmacol. 137 (1), 534-542. doi: 10.1016/j.jep.2011.06.002

Toriumi, Y., Kamei, T., Murata, K., Takahashi, I., Suzuki, N., and Mazda, O. (2012). Utility of Maoto in an influenza season where reduced effectiveness of oseltamivir was observed - a clinical, non-randomized study in children. Forsch Komplementmed. 19 (4), 179-186. doi: 10.1159/000341547

Tran, T. T., Kim, M., Jang, Y., Lee, H. W., Nguyen, H. T., Nguyen, T. N., et al. (2017). Characterization and mechanisms of anti-influenza virus metabolites isolated from the Vietnamese medicinal plant Polygonum chinense. $B M C$ Complement. Altern. Med. 17 (1), 162. doi: 10.1186/s12906-017-1675-6

Wang, X., Yu, S. Y., Shen, P. L., Wang, S. L., Wei, Z. H. , Liu, Z. G., et al. (2017) Analysis of anti-influenza virus pharmacodynamics of QingQiLiangYing granule and its molecular mechanism. Chin. Pharmacol. Bull. 33, 719-728. doi: 10.3969/j.issn.1001-1978.2017.05.025

Wang, C. X., Gao, G. X., Wei, S. C., and Xu, H. R. (2005). [The dynamic effect of yiqi qingwen jiedu heji on the protein expressions of cytokine IFN-gamma, TNF-alpha, IL-10 and IL-6 in the lungs of mice infected by IV FM1]. Zhongguo Zhong Yao Za Zhi 30 (7), 541-544.

Wang, L., Zhang, R. M., Liu, G. Y., Wei, B. L., Wang, Y., Cai, H. Y., et al. (2010). Chinese herbs in treatment of influenza: a randomized, double-blind, placebocontrolled trial. Respir. Med. 104 (9), 1362-1369. doi: 10.1016/ j.rmed.2010.05.015

Wang, C., Cao, B., Liu, Q. Q., Zou, Z. Q., Liang, Z. A., Gu, L., et al. (2011). Oseltamivir compared with the Chinese traditional therapy maxingshiganyinqiaosan in the treatment of $\mathrm{H} 1 \mathrm{~N} 1$ influenza: a randomized trial. Ann. Intern Med. 155 (4), 217-225. doi: 10.7326/0003-4819-155-4-201108160-00005

Wang, Y. G., Jiang, M., Wang, R. B., Zha, Q. L., Zhang, S. J., Zhou, G. Q., et al. (2012). Duration of viral shedding of influenza A (H1N1) virus infection treated with oseltamivir and/or traditional Chinese medicine in China: a retrospective analysis. J. Tradit. Chin. Med. 32 (2), 148-155. doi: 10.1016/ s0254-6272(13)60004-7

Wang, Y., Zhou, B., Lu, J., Chen, Q., Ti, H., Huang, W., et al. (2017). Inhibition of influenza virus via a sesquiterpene fraction isolated from Laggera pterodonta by targeting the NF- $\mathrm{\kappa B}$ and $\mathrm{p} 38$ pathways. BMC Complement. Altern. Med. 17 (1), 25. doi: 10.1186/s12906-016-1528-8

Webster, R. G., Bean, W. J., Gorman, O. T., Chambers, T. M., and Kawaoka, Y. (1992). Evolution and ecology of influenza A viruses. Microbiol. Rev. 56 (1), 152-179. doi: 10.1128/MMBR.56.1.152-179.1992

Xu, H. R., Wang, C. X., Wang, L., Zhou, P. A., Yin, R. Y., Jiang, L. D., et al. (2014). [Mechanism of tonifying Qi traditional Chinese medicines contained in Yiqi Qingwen Jiedu mixture against influenza immune inflammatory injury]. Zhongguo Zhong Yao Za Zhi 39 (20), 4020-4026.

Yakuboğulları, N., Genç, R., Çöven, F., Nalbantsoy, A., and Bedir, E. (2019). Development of adjuvant nanocarrier systems for seasonal influenza A (H3N2) vaccine based on Astragaloside VII and gum tragacanth (APS). Vaccine 37 (28), 3638-3645. doi: 10.1016/j.vaccine.2019.05.038

Yan, Y. Q., Fu, Y. J., Wu, S., Qin, H. Q., Zhen, X., Song, B. M., et al. (2018). Antiinfluenza activity of berberine improves prognosis by reducing viral replication in mice. Phytother. Res. 32 (12), 2560-2567. doi: 10.1002/ptr.6196

Yan, H., Wang, H., Ma, L., Ma, X., Yin, J., Wu, S., et al. (2018). Cirsimaritin inhibits influenza A virus replication by downregulating the NF- $\mathrm{KB}$ signal transduction pathway. Virol. J. 15 (1), 88. doi: 10.1186/s12985-018-0995-6

Yao, J., Zhang, H., Ma, L., Mu, X., Wang, Y., Lu, Y., et al. (2018). Effect of traditional Chinese medicine Bupleurum in the treatment of influenza A (H1N1). Pak J. Pharm. Sci. 31 (4(Special), 1713-1717.

Yin, J., Ma, L., Wang, H., Yan, H., Hu, J., Jiang, W., et al. (2017). Chinese herbal medicine compound Yi-Zhi-Hao pellet inhibits replication of influenza virus infection through activation of heme oxygenase-1. Acta Pharm. Sin. B. 7 (6), 630-637. doi: 10.1016/j.apsb.2017.05.006

Yoshino, T., Arita, R., Horiba, Y., and Watanabe, K. (2019). The use of maoto (Ma-Huang-Tang), a traditional Japanese Kampo medicine, to alleviate flu symptoms: a systematic review and meta-analysis. BMC Complement. Altern. Med. 19 (1), 68. doi: 10.1186/s12906-019-2474-Z

Zhang, T., Xiao, M., Wong, C. K., Mok, K. C., Zhao, X., Ti, H., et al. (2018). Sheng Jiang San, a traditional multi-herb formulation, exerts anti-influenza effects in vitro and in vivo via neuraminidase inhibition and immune regulation. $B M C$ Complement. Altern. Med. 18 (1), 150. doi: 10.1186/s12906-018-2216-7 
Zhi, H., Jin, X., Zhu, H., Li, H., Zhang, Y., Lu, Y., et al. (2020). Exploring the effective materials of flavonoids-enriched extract from Scutellaria baicalensis roots based on the metabolic activation in influenza A virus induced acute lung injury. J. Pharm. BioMed. Anal. 177:112876. doi: 10.1016/j.jpba.2019.112876

Zhou, Z., Li, X., Liu, J., Dong, L., Chen, Q., Liu, J., et al. (2015). Honeysuckleencoded atypical microRNA2911 directly targets influenza A viruses. Cell Res. 25 (1), 39-49. doi: 10.1038/cr.2014.130

Zhou, X., Li, H., Shi, Z., Gao, S., Wei, S., Li, K., et al. (2017). Inhibition activity of a traditional Chinese herbal formula Huang-Lian-Jie-Du-Tang and its major components found in its plasma profile on neuraminidase-1. Sci. Rep. 7 (1), 15549. doi: 10.1038/s41598-017-15733-7

Zhu, H. Y., Han, L., Shi, X. L., Wang, B. L., Huang, H., Wang, X., et al. (2015). Baicalin inhibits autophagy induced by influenza A virus H3N2. Antiviral Res. 113, 62-70. doi: 10.1016/j.antiviral.2014.11.003

Zhu, H., Lu, X., Ling, L., Li, H., Ou, Y., Shi, X., et al. (2018). Houttuynia cordata polysaccharides ameliorate pneumonia severity and intestinal injury in mice with influenza virus infection. J. Ethnopharmacol. 218, 90-99. doi: 10.1016/ j.jep.2018.02.016

Conflict of Interest: The authors declare that the research was conducted in the absence of any commercial or financial relationships that could be construed as a potential conflict of interest.

The handling editor declared a shared affiliation, though no other collaboration, with the authors at the time of the review.

Copyright (C) 2020 Xiong, Li, Duan, Liu, Zhu, Zhang, Li, Lu and Huang. This is an open-access article distributed under the terms of the Creative Commons Attribution License (CC BY). The use, distribution or reproduction in other forums is permitted, provided the original author(s) and the copyright owner(s) are credited and that the original publication in this journal is cited, in accordance with accepted academic practice. No use, distribution or reproduction is permitted which does not comply with these terms. 